\title{
HANDS-ON MEMS: BUILDING COMPETENCE THROUGH PRACTICAL LEARNING EXPERIENCES
}

\author{
L.G. Fréchette ${ }^{1,2}$, C.W. Wong ${ }^{2}$, K. Chin ${ }^{3}$, G. Georgiou ${ }^{3}$, K.F. Farmer ${ }^{4}$, F. Miller ${ }^{5}$, V. Modi ${ }^{2}$ \\ ${ }^{1}$ Université de Sherbrooke, Dept of Mechanical Engineering, Sherbrooke, QC, Canada \\ ${ }^{2}$ Columbia University, Dept of Mechanical Engineering, New York, NY, USA \\ ${ }^{3}$ New Jersey Institute of Technology, Department of Physics, Newark, NJ, USA \\ ${ }^{4}$ TSI Incorporated, Shoreview, MN, USA \\ ${ }^{5}$ Lehigh University, Microelectronics Research Laboratory, Bethlehem, PA, USA
}

\begin{abstract}
Over the past 5 years, we have developed an education program in MEMS with original activities that build student competences in the development of MEMS devices. This paper first presents the underlying philosophy of education adopted here, which focuses on competence as opposed to knowledge. The specific pedagogical activities or tools developed and used for our MEMS course will then be presented, including: lectures, casestudies, virtual microfabrication software, a focused design project with clear goals, and hands-on testing of specially designed MEMS devices.
\end{abstract}

\section{INTRODUCTION}

It is particularly challenging to adequately prepare students in the field of micro-engineering since the development of microelectromechanical systems (MEMS) is both highly multidisciplinary and technology-intensive. Typical MEMS devices can integrate elements traditionally associated with distinct fields of engineering and science, including mechanical, electrical, and chemical engineering, applied physics, chemistry, and biology. For example, the development of a MEMS optical switch may require elements from structural mechanics, electrostatics, material science, circuits, and photonics, while a microfluidic biosensor may involve fluid and mass transport, microelectronics, control theory and molecular biology to name a few. The micro and nanofabrication technologies available to implement such microsystems are typically based on semiconductor manufacturing, which is an important body of knowledge in itself. Novel devices constantly introduce new requirements which push the limits of current fabrication technology, leading to the development of less traditional materials and fabrication approaches.

It is therefore not likely for a student to acquire comprehensive knowledge in all the disciplines used in the design of MEMS and master the broad range microfabrication technologies involved in their implementation. The question is then: what should a MEMS course or program accomplish? If the objective is to prepare the student to undertake the development of MEMS in industrial or academic settings, then the course should aim at raising his level of competence in doing so. In this article, we present our competence-based learning approach for MEMS followed by the curriculum, pedagogical activities, and tools that we have developed to implement it. This work has evolved over the past 5 years through the increasing interaction of faculty members at the New Jersey Institute of Technology (NJIT), Columbia University, Lehigh University, and the Université de Sherbrooke [2].

\section{COMPETENCE-BASED LEARNING}

In defining the content of our MEMS course, we focused on activities that cover the spectrum of skills and knowledge required for the development of MEMS, while minimizing the need for extensive facilities. Generally, competence will be gained by developing [1]:

1) scientific or disciplinary knowledge;

2) technical skills or aptitudes;

3) personal and interpersonal skills or attitude.

Development of the student's aptitudes and attitude are equally important as scientific knowledge, given the innovationdriven nature of MEMS. The students should develop competence to pursue the following steps: Invention - Design Implementation - Operation. The competencies required to realize these four steps go beyond disciplinary knowledge, to include aptitudes and attitude, as illustrated in Figure 1. In class, these are implemented through a combination of: lectures, homeworks and examinations, case studies, hands-on MEMS laboratories, virtual microfabrication and a team design project. The contributions of these activities to the various skills and attitudes to be developed are listed in Table 1. The following sections will describe the overall course curriculum, followed by the main activities: Handon MEMS testing laboratories, ICLAB virtual microfabrication software, and team design projects.

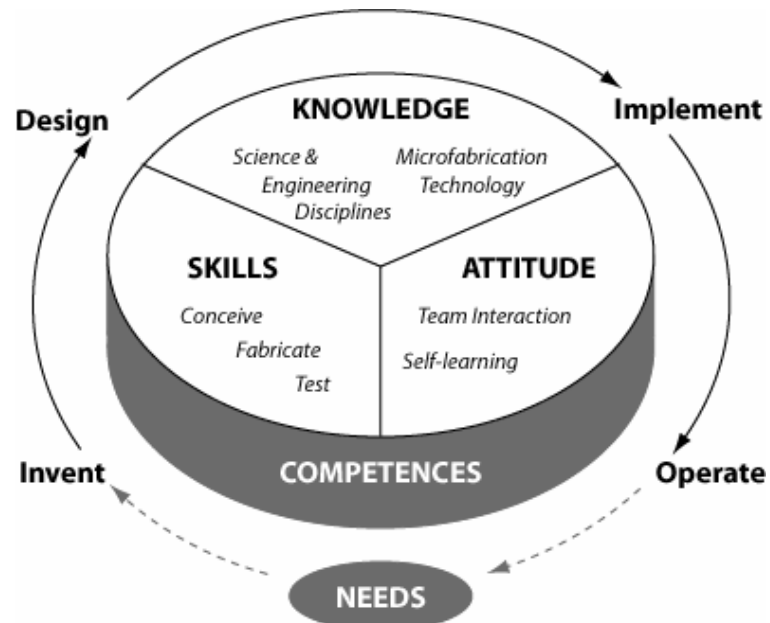

Figure 1 - Competency-oriented MEMS education: the underlying philosophy that guides the choice of pedagogical activities for the MEMS course. 


\section{COURSE CURRICULUM}

The activities used in our MEMS courses have evolved over the past years, and have not necessarily all been used simultaneously. The course is aimed towards new graduate students or engineering seniors. Typically, the first half of the semester focuses on lectures to provide an introduction to 1) MEMS markets, applications, and scaling; 2) MEMS materials and fabrication methods, with emphasis on silicon micromachining and process integration; 3) MEMS operating and transduction principles including: mechanics, dynamics, electrostatics, piezoresistance, piezoelectricity, and thermo-mechanics; 4) MEMS device concepts, for pressure, acceleration, and rotation, as well as more advanced devices; 5) Microfluidics: Flow, heat and mass transfer at small scales; electrokinetics; power MEMS and bioMEMS; 6) Photonics and optical MEMS; 7) MEMS device design, simulation tools, and characterization techniques, and MEMS packaging concepts. Case studies are used to contextualize and integrate the material presented. Individual homework is mainly used for evaluation, as well as a mid-term exam. The main textbook used for the course is that of Senturia [3], but the students are also recommended to consult Kovacs [4] as necessary.

The second half of the time in class is devoted to more practical activities, during which the students will gain hands-on experience in testing MEMS sensors and actuators specifically designed for educational purposes, design a MEMS device, and familiarize themselves with microfabrication with a unique cleanroom simulation software. The following sections will describe these activities.

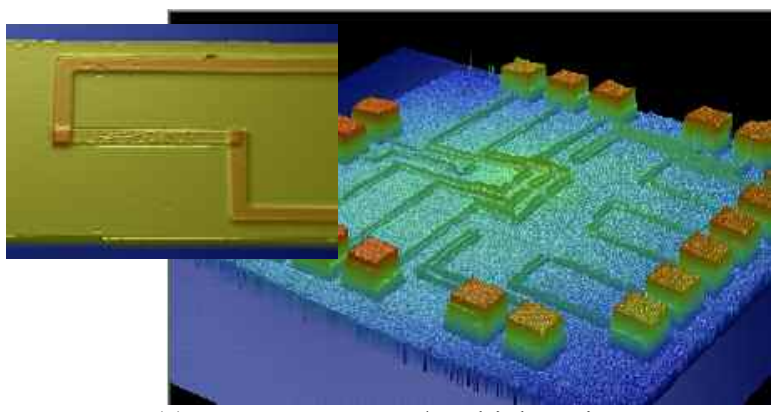

(a) Pressure sensor $\mathrm{w} /$ multiple resistors

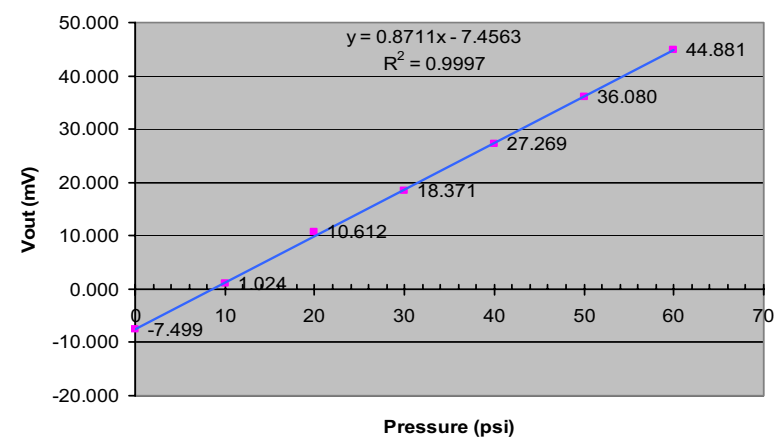

(b) Wheatstone bridge output versus pressure

Figure 2 - Piezoresistive pressure sensors used for the hands-on test laboratories, specially designed for educational purposes: a) Optical profilometer image of the various piezoresistors located on the membrane, showing one resistor in insert; (b) Experimental measurements by the students at Columbia, showing the Wheatstone bridge output as a function of applied pressure for selected resistances.

\section{HANDS-ON MEMS LABORATORIES}

In the hands-on laboratories, two MEMS devices are tested: 1) a piezoresistive pressure sensor; and 2) an electrostatic actuator. They were specially developed for educational purposes by Prof. K. R. Farmer at NJIT [2].

The pressure sensor shown in Fig. 2 consists of a single crystal silicon membrane formed by anisotropic wet etching and has multiple diffused strain gauges (piezoresistors) distributed over the membrane. The students can first perform individual measurements of resistance as a function of the applied pressure. The stress distribution over the membrane, as determined by the various piezoresistors, can be compared to analytical and finite element solutions in order to better understand the structural mechanics involved. Various Wheatstone bridge configurations are then evaluated to highlight the importance of circuits in transduction (sample results are shown in Fig. 2b).

The electrostatic actuator is a micromirror attached by a torsionnal spring, as illustrated in Fig. 3a. It is formed by deep reactive ion etching of a thin $\mathrm{Si}$ wafer (50 microns) that is bonded over a pit in a thick silicon substrate. An oxide layer between the wafers allows the application of a potential between the mirror and the substrate. The resulting electrostatic force pulls the mirror down and makes it rotate about the torsionnal springs. Measurements of deflection are simply taken by tracking a light beam that is reflected off the tilting mirror as a function of applied voltage (Fig. 3b).

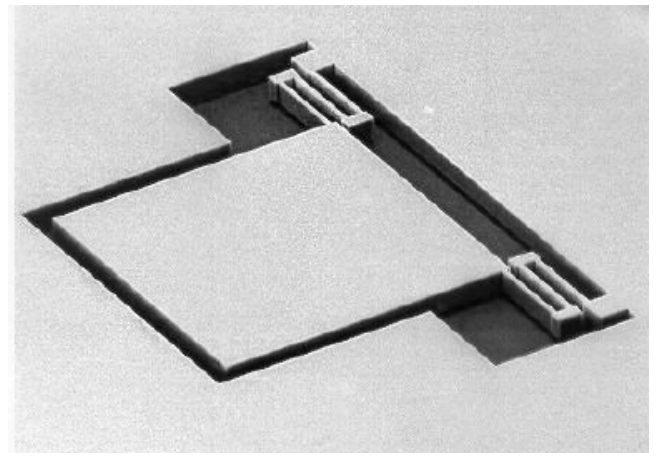

(a) Electrostatic micromirror

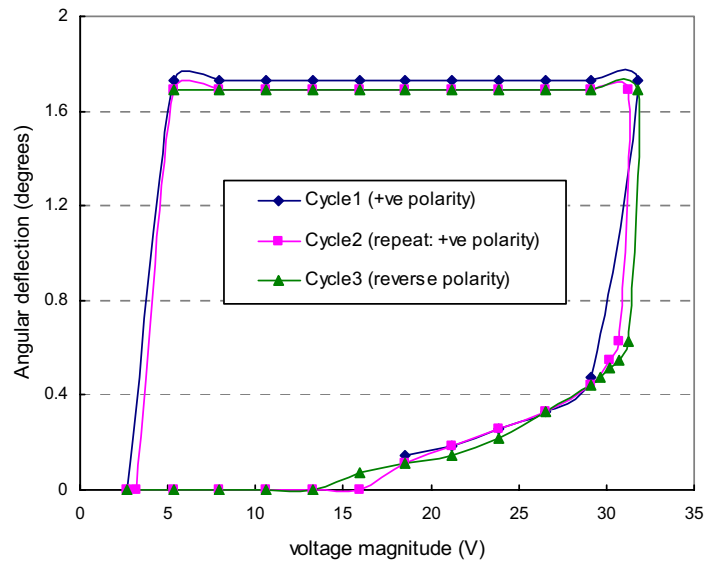

(b) Angular displacement versus DC voltage

Figure 3 - Electrostatic torsion micromirror (actuator) used for the hands-on test laboratories. It allows investigation of electrostatic actuation, including pull-in, hysteresis, and even fluidic damping (dynamic response tests, not shown here). 
In this laboratory, the students have the opportunity to explore the non-linear behavior of electrostatic forces and the pull-in instability phenomena. When comparing the results to simple analytical solutions, the students must explain why the mirror remains down even when the applied potential is lowered below the pull-in value. This pushes the students to better understand the balance between the forces involved and the origin of the pull-in instability. Dynamic response testing was also conducted with naturally underdamped and overdamped devices to illustrate the behavior of this second order system and introduce the concept of viscous damping. These measurements were simply done with a function generator in open air, since a sufficient range of damping ratios can be achieved through changes of the geometry.

Throughout these laboratories, the students develop their experimental skills and procedures, learn to interpret and analyze experimental data, while stimulating their curiosity and excitement for MEMS. Unlike commercial MEMS, these educational test devices are not completely packaged so students can manipulate the chips and directly measure their dimensions. The presence of superfluous piezoresistors or multiple geometries further enhance the learning experience. Relatively simple instrumentation is used such that these laboratories could be implemented at other institutions with minimal investment.

\section{VIRTUAL MICROFABRICATION}

To familiarize the students with cleanroom equipment and processing, a virtual microfabrication environment is used: ICLAB by F. Miller [5]. Unlike commercial MEMS software (Coventor, Intellisuite), ICLAB simulates the consecutive processes in real time (or faster), requires control of the process equipment through schematics while respecting realistic constraints, and allows students to see their wafer cross section as it is etched or films deposited (Fig. 4a).

For example, Fig. $4 \mathrm{~b}$ displays a virtual sputter-etching system. The student must operate the system by first loading a wafer into the vacuum chamber. Next the pump is started and the air pumped out of the chamber. Valves are opened to allow selected gases to flow into the chamber. Flow meters must be set to adjust gas flow and chamber pressure. Power is turned on and set to the desired level. Etching will now start but only if the pressure is within the correct range, a wafer is actually in the chamber and the combination of films on the wafer and etch gas would actually allow etching to occur.

As the etch proceeds, the student will be able to watch the etching progress by viewing a cross section of the wafer. A basic spectrometer is available to assist with endpoint detection. Etching is stopped by turning off power. If the student does not turn off power etching will continue until everything that can etch is removed from the wafer (including the wafer).

All of the individual processes available in ICLAB operate in the same manner as the plasma etching described above. They include oxidation, ion implantation, lithography, chemical etching, CVD, PVD, CMP, and wafer cleaning. Limited backside processing was added to allow MEMS processing. Students carry out individual process steps and immediately see the results of their action. Students can feel free to explore the virtual processing environment where mistakes provide valuable learning experience but cost nothing in terms of lost materials, lost lab time, or student safety.

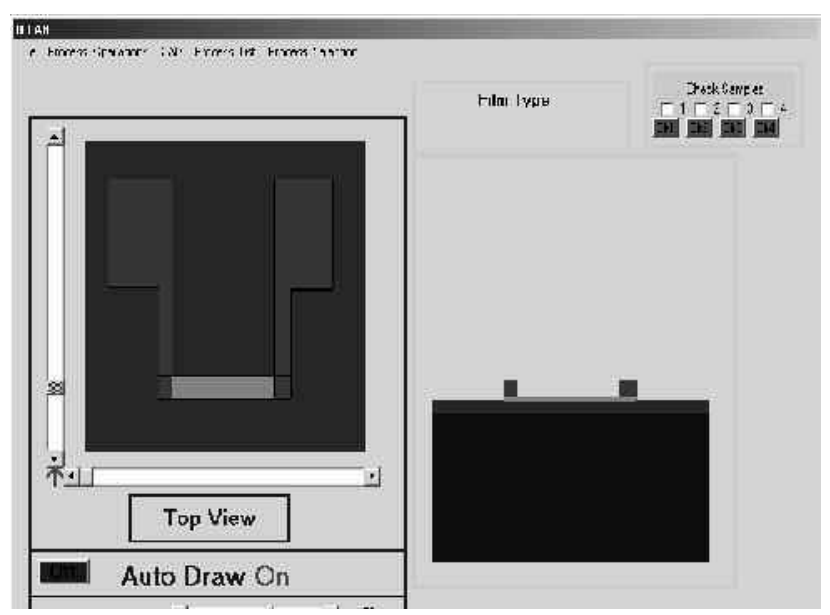

(a) Top and cross section view of wafer

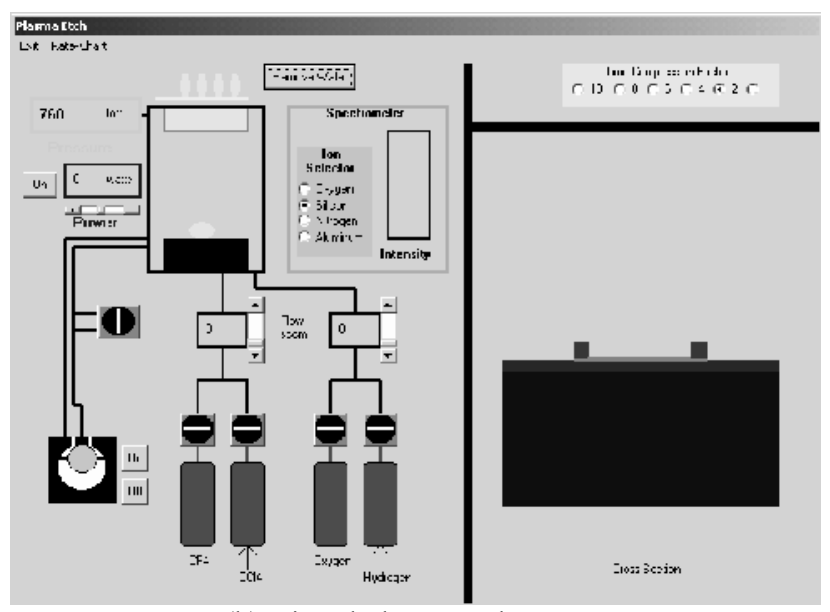

(b) Virtual plasma etch system

Figure 4 - ICLAB: a virtual cleanroom processing environment that allows the students to de-mystify cleanroom processing and microfabrication.

The use of virtual fabrication software allows hands-on microfabrication experience, without the cost, time, and infrastructure requirement associated with real cleanroom experience. It also helps the students appreciate the challenges related to process integration, such as thermal budgets, selectivity, and materials compatibility. This software is mostly used to complement the lectures on microfabrication, but has also been used for case studies and design projects.

\section{TEAM DESIGN PROJECTS}

The initial portion of the cycle shown in Fig. 1 is implemented with a MEMS design project. It starts either from a specific need or from an original idea. The students must invent and design a MEMS device that addresses the need or that implements the idea. At Sherbrooke, the students first translate the needs into requirements and specifications, and then undertake the design of a novel MEMS device to meet them. At Columbia, the topics are proposed and supported by faculty members. This approach has been fruitful, since some projects have led to further research and development as thesis projects, based on innovative directions from the students themselves.

Teams are typically formed of students with different backgrounds to favor multidisciplinary interactions and cover the 
range of disciplines required for a project. During the design process, the students need to deepen their knowledge beyond the level covered in class in one or more disciplinary areas. They must also define a viable process flow for their device, which often proves to be very challenging without expert assistance. Faculty or graduate mentors therefore play an important role in the quality of the projects. Students have consistently remarked that they learnt more during the actual design project process.

A final group report and presentation is scheduled, with a panel of faculty judges. Throughout the activities in the second half of the semester, the students develop their competencies for MEMS design, fabrication, and characterization while improving their knowledge of the physics and fabrication processes used in the development of MEMS.

\section{DISCUSSION AND CONCLUSIONS}

The pedagogical activities and tools presented above have been very well received by the students, based on their end-of-term course evaluations and comments. Enrollment has continuously increased, going from 16 students in 2002 to 37 students in 2005 (Columbia). Although the majority of students have been from mechanical engineering (avg. 80\%), there has been students from electrical engineering, applied physics, biomedical engineering, civil engineering, and industrial engineering.

Although the competence-based learning approach with hands-on testing, virtual microfabrication and project projects would benefit from further development, these activities appear to be well suited for teaching MEMS. Since MEMS is inherently a device or product oriented field, it is amenable to practical, hands- on activities. Unfortunately, MEMS technology is less accessible due to the cost of cleanroom infrastructures and the lack of MEMS devices dedicated for education. Through the work presented here, the objective has been to alleviate some of these limitations to make hands-on MEMS education widely accessible. Portions of this work have been supported by NSF 02-043; this support is gratefully acknowledged.

\section{REFERENCES}

[1] Proulx, D., Brouillette, M., Charron, F., Nicolas, J. "A New Competency-Based Program For Mechanical Engineers",

Presented at the CSME Forum 1998, Ryerson Polytechnic Univ., Toronto,19-22 May 1998.

[2] Fréchette, L., Modi, V., Miller, F., Farmer, K.R., "Hands-on MEMS", 15th Biennial University/Government/Industry

Microelectronics Symposium, Boise, Idaho, June 30 - July 2, 2003.

[3] Senturia, S. D., Microsystem Design, Kluwer, Boston, 2001.

[4] Kovacs, G., Micromachined Transducers Sourcebook, McGraw Hill, New York, 1998.

[5] Miller, F., "Virtual Reality in Microelectronics Laboratory Instruction," Proc. of the 14th Biennial University/Government /Industry Microelectronics Symp. Richmond, VA, June 18-20, 2001.

Table 1 - List of competencies developed in the MEMS course with the pedagogical activities. Stars $(*$ or $* *)$ indicate the level of contribution of each activity to the skill or attitude developed.

\begin{tabular}{|l|c|c|c|c|c|c|}
\hline \multicolumn{1}{|c|}{ SKILLS / APTITUDES / KNOW-HOW } & $\begin{array}{c}\text { Lectures } \\
\text { homework, } \\
\text { reading }\end{array}$ & $\begin{array}{c}\text { Case } \\
\text { Studies }\end{array}$ & $\begin{array}{c}\text { Design } \\
\text { Project }\end{array}$ & $\begin{array}{c}\text { Hands- } \\
\text { on Test } \\
\text { Labs. }\end{array}$ & $\begin{array}{c}\text { ICLAB } \\
\text { virtual } \\
\text { fab. }\end{array}$ & $\begin{array}{c}\text { Clean- } \\
\text { room } \\
\text { fab. }\end{array}$ \\
\hline Define needs & & $* *$ & $*$ & & & \\
\hline Identify potential applications for MEMS & $*$ & $* *$ & $*$ & & & \\
\hline Conceive a device: principles and layout & $*$ & & $* *$ & & & \\
\hline Develop a microfabrication process flow & $*$ & $*$ & $* *$ & & $*$ & $*$ \\
\hline Design the device quantitatively & $* *$ & & $* *$ & & & \\
\hline Analyze the device behavior & $* *$ & $*$ & $*$ & & & $*$ \\
\hline Fabricate the device & & & & & $*$ & $* *$ \\
\hline Develop and integrate microfab. processes & $*$ & & & & $*$ & $* *$ \\
\hline Characterize the device and fab. results & & & & $* *$ & & $* *$ \\
\hline Package the MEMS device & & $*$ & & $* *$ & & \\
\hline Test the device performance & & & & $* *$ & & \\
\hline Integrate the MEMS in an application & & $*$ & $*$ & $*$ & & \\
\hline
\end{tabular}

\begin{tabular}{|c|c|c|c|c|c|}
\hline ATTITUDE & & & & & \\
\hline Self-learning from the literature & $* *$ & $*$ & $*$ & & \\
\hline Interaction with people in other disciplines & & & $* *$ & $*$ & $*$ \\
\hline Work in teams & & & $* *$ & $* *$ & \\
\hline Communicate effectively & & & $*$ & $*$ & \\
\hline Proper cleanroom behavior & & & & & $* *$ \\
\hline Maintain a $\log$ book of R\&D activities & & & $*$ & $*$ & $*$ \\
\hline
\end{tabular}

${ }^{1}$ NOTE: Cleanroom fabrication was not an activity in the current MEMS course at Columbia or Sherbrooke, but a tour of the cleanroom is included to strengthen the learning process. 